\title{
Prosthodontic and comprehensive treatment of temporomandibular disorders
}

\author{
Andreas, Dara Aidilla, Ricca Chairunnisa \\ Department of Prosthodontics \\ Faculty of Dentistry, Universitas Sumatra Utara \\ Medan, Indonesia \\ Corresponding author: Dara Aidilla, E-mail: daradent88@gmail.com
}

\begin{abstract}
Temporomandibular disorder (TMD) is the common term used to describe a range of disorder affecting the temporomandibular joint (TMJ). Facial pain is a common symptom that patients present to their dentist, and TMDs represent a significant proportion of the non-dental causes of this pain. These disorders can have a profound effect on a patient's quality of life. The management goals for the prosthodontist are patient comfortable, occlusal stability and the complex restoration of the teeth.
\end{abstract}

Keywords: temporomandibular joint, temporomandibular disorder, TMD treatment, occlusal appliance

\section{INTRODUCTION}

Temporomandibular disorder (TMD) is a general term used to explain various disorders that affect the temporomandibular joint (TMJ). Facial pain is the most common symptom that often occurs in patients when patients come to the clinic, and pain in TMJ disorders is caused by non-dental. This disorder can have a considerable effect on the quality of life of patients. ${ }^{1}$

Patients who have TMD can present with a range of different symptoms; these include diffuse pain-typically intermittent in nature and often reported in several areas of the head and neck; tenderness or pain in muscles of mastication; limited mouth opening and locking of the jaw; painful of teeth (wear facets may be noted on tooth surfaces); and clicking or crepitus sounds when TMJ is in function. ${ }^{1}$

Therapeutic approaches to the TMJ disorders must be directed to reduce the main signs and symptoms of the condition. In the majority, patients who have disorders in the TMJ must be encouraged to be able to carry out early self-management that can help control symptoms and limit functional disorders. However, pain is the main cause of this pathological state, and this is the main reason patients seek medical treatment. ${ }^{1,2}$

Conservative treatments for TMD include medication, physiotherapy, occlusal splints, self-management strategies, and interventions based on cognitive behavioral approach. At present, a conservative treatment approach prevails over surgery, given it is less aggressive and usually result in satisfactory clinical outcomes in the mild-moderate TMD. In fact, the evidence for the greatest effectiveness of surgical versus conservative intervention to reduce short-term pain in arthrogenic TMD is controversial and inconclusive. ${ }^{2}$

Treatment of TMD begins with anamnesis and a thorough examination. Signs and symptoms in TMD can generally be directly identified; however, it is important to pay attention to other diagnoses that can resemble or occur simultaneously. There are certain signs \& symptoms that are categorized into "red flag". 3

Certain signs and symptoms that are categorized into "red flag" need to be considered and if found, they must be all the way to refer to more competent practitioners. It must be noted that the symptoms which are a red flag category have a special type of examination and a different treatment than generally. When the signs and symptoms found are not categorized into red flag, treatment can be done conservatively. ${ }^{3}$

Majority of patients with TMD need to be given non-invasive handling that can relieve symptoms rather than patients. TMD almost resemble rheumatological disorders; and it is still unknown to the cause rather than TMD so that the signs and symptoms can be more severe. So, it is necessary to be given direct handling to avoid invasive and irreversible treatments. ${ }^{4}$

So, it is very urgent to understand prosthodontic and comprehensive treatment of temporomandibular disorders.

\section{CONSIDERATIONS IN THE TMD}

Accurately diagnosing and treating TMDs can be a difficult and confusing task. This is often true primarily because patient's symptoms do notalways fit into one classification. All the treatment methods being used for TMDs can be categorized generally into one of two types, namely definitive treatment or supportive therapy. Definitive treat- 
ment refers to those methods that are directed toward controlling or eliminating the etiologic factors that have created the disorder. Supportive therapy refers to treatment methods that are directed toward altering patient symptoms but often do not affect the etiology. ${ }^{5}$ Definitive therapy is aimed directly toward the elimination or alteration of the etiologic factors that are responsible for the disorder. Definitive treatment for an anterior displacement of the articular disc will reestablish the proper condyle-disc relationship. Since it is directed toward the etiology, an accurate diagnosis is essential. An improper diagnosis leads to improper treatment selection. ${ }^{5}$

Supportive therapy is directed toward altering the patient's symptoms and often has no effect on the etiology of the disorder. A simple example is giving a patient aspirin for a headache that is caused by hunger. The patient may feel relief from the headache, but there is no change in the etiologic factor (hunger) that created the symptom. Supportive therapy is directed toward the reduction of pain and dysfunction. The two general types of supportive therapies; they are pharmacologic therapy and physical therapy. ${ }^{5}$

Durhametal categorize some signs and symptoms in orophacic pain, which can resemble TMD. This category is referred to as "red flag" covers 1) previous history of malignancy-could indicate new primary, recurrence, or metastases; 2) persistent or unexplained neck lump or cervical lymphadenopathy-may indicate a neoplastic, infective, or autoimmune cause; 3 ) neurological symptoms, for example, headache or cranial nerve abnormalities with sensory or motor function changemay indicate an intracranial cause, or malignancy affecting cranial nerve peripheral branches; 4) facial asymmetry, facial swelling, or profound trismus-may indicate a neoplastic, infective, or inflammatory cause; 5) recurrent epistaxis, purulent nasal discharge, persistent anosmia, or reduced hearing on the ipsilateral side-may indicate nasopharyngeal carcinoma; 6 ) unexplained fever or weight loss-may indicate malignancy, immunosuppression, or other infective causes, for example, septic arthritis; 7) new-onset unilateral headackelor scalp tenderness, jaw claudication, and general malaise, if the person is more than 50 years of age-may indicate giant cell arteritis;and 8)occlusal changes-mayindicateneoplasiarheumatoid arthritis, trauma, or bone growth around the TMJ, for example, acromegaly. ${ }^{1}$

Signs and symptoms categorized into "red flag" need to be considered and if found, they must be all the way to refer to more competent practitioners. It must be noted that these red flag symptoms in isolation should prompt their own line of enquiry and management. When red flags have been considered and confidently excluded, the mainstay of treatment for majority of people is conservative. ${ }^{3}$

Treatment for TMD is categorized into two types namely definitive care and supportive care. But it mustalways be remembered that this supportive treatment is only symptomatic and not a substitute for definitive care. The etiological factors need to be addressed and eliminated so that long-term care success will be achieved. ${ }^{5}$

\section{TREATMENT OF TMD}

The main goals of treatment for TMD are to reduce or eliminate pain and or joint noises, and to restore normal mandibular function, that is best achieved when other contributing factors such as stress, depression and oral parafunctional habits (ie., bruxism) are addressed and incorporated into the overall treatment strategy. It is essential for the clinician to establish whether the fundamental problem is physical or psychogenic, as this will dictate treatment. ${ }^{6}$

\section{Explanation and reassurance}

The initial step in the management of TMD is to explain to patients the causes and nature of the disorders they faced, then convince them of the benign nature of the situation. It must be explained to patients that this disorder is not life-threatenening for the patient, not like cancer, and but this disorder can be chronic in nature. Then explain to patients this TMD can be treated. ${ }^{6}$

The initial step in the management of TMD is to explain to patients the causes and nature of the disorders they faced, then convince them of the benign nature of the situation. We must explain to patients that this disorder is not life-threatening for the patient, not like cancer, and but this disorder can be chronic in nature. Then explain to patients this TMD can be treated. ${ }^{6}$

\section{Education and selfcare}

The next step is to formulate a self-care routine which should include the following; limitation of mandibular function, habit awareness and modification, a home exercises program and avoiding stress. Voluntary limitation of mandibular function (i.e., avoid excessive chewing and talking) is encouraged to promote rest or the immobilisation of muscular and articular structures, much the same 
as an athlete would rest an injured joint. Hence, the patient is advised to maintain a soft diet and avoid foods where a considerable amount of chewing is involved; ${ }^{6}$ 1) soft diet: existing pain in the mastication system often limits functional range of mandibular movements. Therefore, if possible, painful movements should be avoided. In addition, this should be avoided because it can improve symptoms of the disorder through the effects of central excitatory and cyclic muscle pain. Patients are given instructions to move their mandible in a range that does not provide pain. It means that the type offood must be changed. Patients are encouraged to eat softer diet, bite less, and generally chew slowly (Fig.1);5 2)jaw rest (especially in long dental

\begin{tabular}{|c|c|}
\hline \multicolumn{2}{|l|}{ 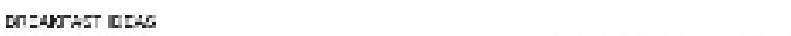 } \\
\hline $\operatorname{vadet} \ldots$ ave & 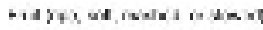 \\
\hline 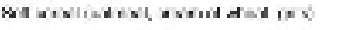 & etanis \\
\hline Dgai laxead wop nat: & Deagga tann: \\
\hline \multicolumn{2}{|l|}{ 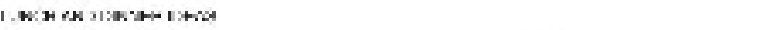 } \\
\hline 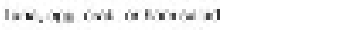 & $\mathrm{x}=$ \\
\hline 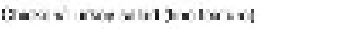 & $\operatorname{lan} 0$ \\
\hline 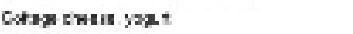 & Futs \\
\hline Dan agoviting & bascos \\
\hline Was $=1,0 \times 1 \times$ & $\omega$ \\
\hline 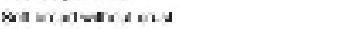 & 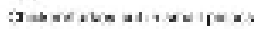 \\
\hline 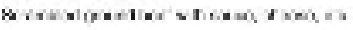 & Nonswn \\
\hline $\begin{array}{l}\text { Reap iv dine } \\
\text { rah, ativira pase }\end{array}$ & 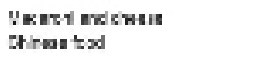 \\
\hline \multicolumn{2}{|l|}{ Itosan n:tion } \\
\hline ka azun & Tutalr; \\
\hline HED & Eesp ked \\
\hline Aritag tared & Ersotwa ribition \\
\hline \multicolumn{2}{|l|}{ patzik to nne: } \\
\hline 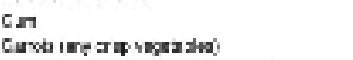 & 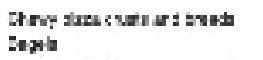 \\
\hline 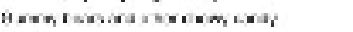 & 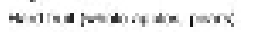 \\
\hline lavs & 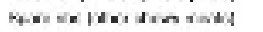 \\
\hline nown & 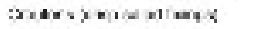 \\
\hline Otha raxy ex:ann & 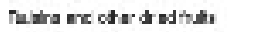 \\
\hline 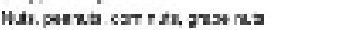 & fexse sunct fetsa \\
\hline
\end{tabular}

Figure 1 Suggested patient education form for instructtions on a soft food diet. ${ }^{5}$

treatments): after checking hard tissue and initial handling, soft tissue in the joints also needs to be evaluated. Treatment in soft tissue will increase according to the severity of the symptoms. If there is no significant change in the range of movements and slight pain, the patient is asked to reduce use, start a soft diet and generally rest the jaw for two weeks. Patients should be reminded not to chew gum and if possible, reduce parafunctional activity. Patients are instructed to return to clinic if there is an increase in pain and an appointment is made for re-evaluation within 2 weeks; ${ }^{5} 3$ ) avoid excessive jaw movements: Patients must also be instructed not to evaporate widely, sing, chew gum, and other activities that can improve the function of lower jaw movements; ${ }^{5} 4$ ) topical heat and coolant therapy: The modality of physical therapy must be done carefully because manipulation of therapy can increase pain. Sometimes stimulation of hot sensations can help. In some patients, cold sensations are more helpful. Patients themselves can know which is better at reducing their pain. Like warm therapy, cold therapy has proven to be a simple and often effective method for reducing pain. Being subjected to a cold sensation can relax the muscles that are spasms, thereby reducing pain. Applicationis carried out directly into the pain area and transferred by a circular motion without emphasis. When numb starts to feel, stop immediately and do not leave on the tissue for more than 5 to 7 minutes. After a period of heating and cooling, a second application may be needed (Fig.2);55)avoid

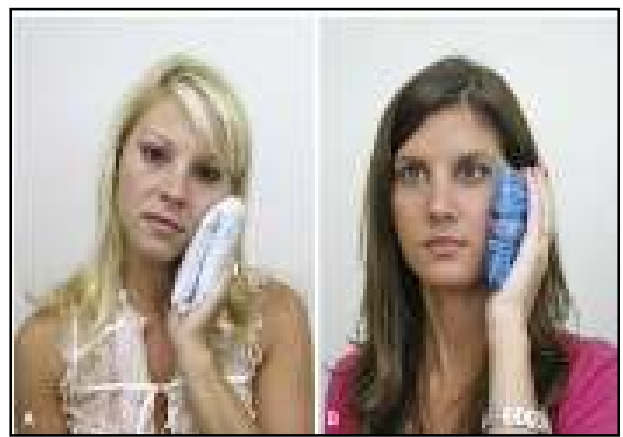

Figure 2 Heat and coolant therapy. ${ }^{5}$

stress and anxiety: Some TMD are etiologically related to certain emotional states. The emotional stress is certainly one of several psychological factors that need attention. Increased levels of emotional stress can affect muscle function by increasing rest activity (protective co-contracting), increasing bruxism or both. Increased emotional stress also activates the sympathetic nervous system, which itself can be a source of muscle pain. One anxiety disorder that might be considered the most difficult by clinicians is obsessive compulsive disorder. This disorder has been shown to increase TMD in patients. ${ }^{5}$

\section{Medicament}

Anti-inflammatory can be used when clinicians suspect tissue inflammation such as with capsulitis, retrodiscitis, or osteoarthritis. This type can suppress the body's overall response to irritation. Anti-inflammatory can be given orally or by injection. The main class of oral anti-inflammatory drugs is nonsteroidal anti-inflammatory drugs (NSAIDs). This drug is effective for mild to moderate inflammatory conditions and postoperative acute pain (Fig. 3$)^{5}$

When high levels of emotional stress are considered to contribute to TMD, anxiolytic agents (antianxiety) can help in managing symptoms. Remember that the anxiolytic agents do not relieve 
stress but only change the patient's perception or reaction to stress. Therefore, the use of anxiolytic agents is a supportive treatment. The commonly used anxiolytics group is benzodiazepines. Can be prescribed every day but, there is a possibility for dependency, it should not be used for more than 7 consecutive days (Fig.4). ${ }^{5}$

Muscle relaxants have been prescribed to patients with TMD for years although most clinicians agree that their effectiveness is minimal. Most of the muscle relaxants have a central effect, which soothes the patient. Perhaps this sedation is the main explanation of the positive response for some patients. ${ }^{5}$

Although tricyclic antidepressants were originally developed for depression management, the latest developments from selective serotonin reuptake inhibitors (SSRIs) have proven to be far more effective. ${ }^{5}$

\section{Jaw physiotherapy}

Massage and stretching; deep massage can be more useful than gentle massage in restoring normal muscle function. However, this deep massage must be carried out by a physical therapist. Deep massage itself can help mobilize tissue, increase blood flow to the area, and eliminate trigger points. To increase the effectiveness of deep massage, patients should be given heat stimulation 10-15 minutes before starting the massage. Deep massage tends to relax muscle tissue, and reduce pain (Fig. $5 \mathrm{~A}){ }^{5}$
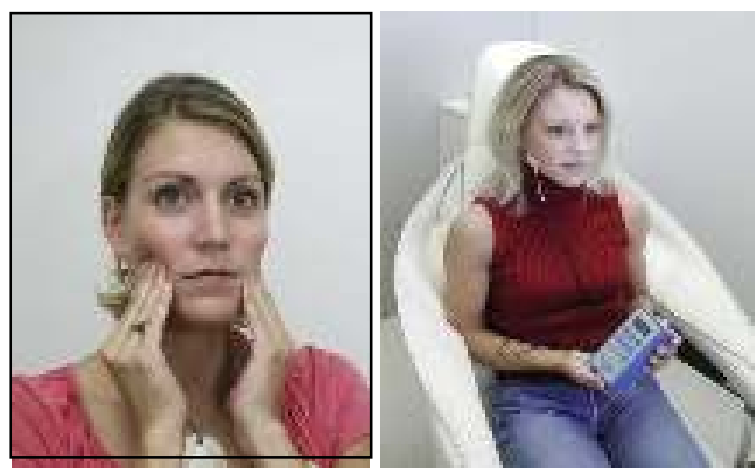

Figure 5A Massage therapy; B TENS therapy. ${ }^{5}$

Transcutaneous electrical nerve stimulation (TENS), produced by continuous stimulus from the nerve fibers at mild pain levels. When TENS units are placed in areas of painful tissue, electrical activity reduces pain perception. TENS uses low voltage, low ampheres, biphasic currents with varying frequencies and is designed primarily for sensory counter stimulation of painful disturbances (Fig.5B). ${ }^{5}$

\begin{tabular}{|c|c|c|c|c|}
\hline Tpe of /ntinfarmatery & Gaverk Kume & Thoda Karte: & Ausreqe Dally Dese. & Masrum Dalf Doet \\
\hline Poxiturel & soderivetwer: & Thend & $325-1000$ reqn & $\begin{array}{l}\text { Potes } \\
\text { 4avat }\end{array}$ \\
\hline \multirow[t]{2}{*}{ Sabcieses } & asotilateyk: & exphin & 325-esorican & 4 grap \\
\hline & Ines: & Dartes & Do-SEbire idd & 1 stemetar \\
\hline \multirow[t]{8}{*}{ hagate ietedrivstins } & taprabst & Main abla & 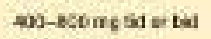 & ssiemeitay \\
\hline & tusoart & Naroen & $250-500$ mplit & 1500 rodsy or 1 - dess \\
\hline & easvocisu soden & Auproa & $275550 \mathrm{~ms}$ obt & 1ebe motayot i f dess \\
\hline & sroperiter & Orids & $30-506 \pi n=4$ & ace resustor 2 wees \\
\hline & seprash & Daporo & $600-1260$ roldes & $1400 \pi$ noty \\
\hline & nolsowen & Nase: & $75-15 \mathrm{madsy}$ & 15 meisy \\
\hline & ebodote & twent & $300-5 \times 0 m e$ bst & $10 c 0$ mplxy \\
\hline & ticriver & Wolnen & $25-10 \mathrm{~ms}$ th & nobiresty \\
\hline Dydowpprese-2 Hertire & polvast & Cotores & $102-250$ rre dit er bes & Acomenty \\
\hline Contonstarid & methipoodisxibert & Nerdo Doserss & $4 m \mid x \in d y s$ & tydidy te dree is decrssse? \\
\hline mase & wrovsae twontherine & Trower & tacsa $4+6 h$ & $\begin{array}{l}40 \text { meiay } \\
\text { So rove then bdys }\end{array}$ \\
\hline
\end{tabular}

Figure 3 Common anti-inflammatory medications used for TMD. 5

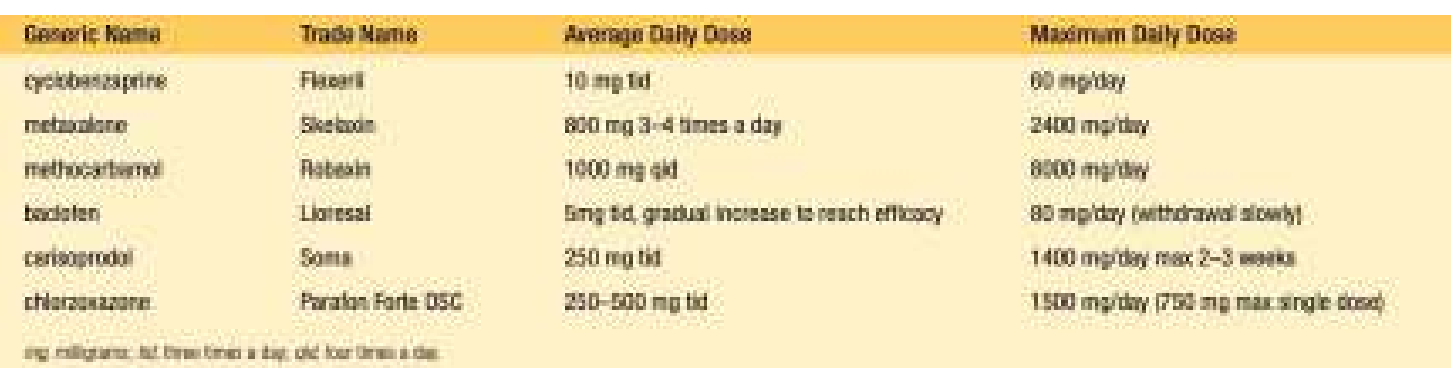

Figure 4 Common muscle relaxants used for temporomandibular disease. ${ }^{5}$ 


\section{Low-level laser therapy (LLLT)}

Among the various physical therapy modalities, LLLT has recently been put under the spotlight because of its easy application, short treatment time, and few contraindications. Many prospective clinical trials have been performed to evaluate the efficacy of LLLT. In addition, LLLT can also improve functionality rather than $\mathrm{TMJ}^{7}$

The use of LLLT has been considered as a complement to treatment options in handling TMD which is maintained to provide responses that resemble analgesics, anti-inflammatory, and regenerative effects, without any side effects being reported and can be well received by patients. ${ }^{7}$

During TMD treatment with LLLT, laser type variability, frequency, dosage, exposure time, application area, number of laser sessions, and duration of therapy can increase the heterogeneity of the treatment effect. However, research is needed to look at different laser parameters of type, treatment regimen, evaluation time, and outcome measures so that they can be used better. This treatment is maintained as noninvasive, safe, easy to use, and inexpensive. ${ }^{7}$

\section{Occlusal appliance therapy}

Occlusal appliance can also help in reducing certain etiological factors. When malocclusion is suspected as a cause of TMD, occlusal appliance therapy can quickly and reversibly produce more desirable occlusal conditions. If it does not affect the symptoms, malocclusion is most likely not an etiological factor and of course the need for irreversible occlusal therapy must be questioned. The success or failure of occlusal appliance therapy depends on the selection, fabrication, and adjustment of the patient's tools and cooperation. ${ }^{5}$

The reversible occlusal therapy temporarily changes the patient's occlusal condition and is best done with occlusal appliance. An acrylic device used on teeth in one of the jaws to create and change position of the mandible and tooth contact patterns. The exact position and occlusion of the mandible will depend on the etiology of the disorder. If you want to treat parafunctional activity, the appliance will provide mandible and occlusion positions that meet the criteria for optimal occlussal relations. So, when the appliance is being used, the occlusal contact patterns are in harmony with the optimal condyle-discus-fossa relationship for the patient. Therefore, this appliance provides orthopedic stability. This tool has been used to reduce symptoms associated with various TMD as well as reduce parafunctional activity. ${ }^{5}$
Irreversible occlusal therapy is a permanent treatment in changing occlusal conditions and or mandible positions, for examples selective gritting of teeth and restorative procedures that change occlusal conditions. Appliance designed to permanently change mandibular growth or repositioning is also considered irreversible occlusal therapy. When treating patients, one must always pay attention to complexity rather than TMD. Therefore, reversible therapy is always indicated as an initial treatment for TMD patients. The success or failure of this treatment can help determine the need for irreversible occlusal therapy. If the patient successfully responds to reversible occlusal therapy (stabilization tool), there seems to be an indication that irreversible occlusal therapy can also help. ${ }^{5}$

\section{Types of occlusal plane}

Stabilizing splint is one of the most-commonly-used splints. Also called Michigan splint, muscle relaxation appliance or gnathologic splint and it's usually made for upper arch. This splint has least adverse effect. ${ }^{8}$

This stabilization can take the form of wearing full arch nighttime occlusal protective devices for patients who have grinding, clenching, or bruxism habits that erode teeth or imperil prosthetic restorations. ${ }^{9}$ Outcome of stabilizing splint also depends on certain factors among which only continuous use of this splint can cause reduction in symptoms of TMD. As concluded by results wearing splint for 24 hours per day results in occlusal stabilization. ${ }^{8}$

Stabilization appliance can help minimize forces to damaged tissues and permitting more efficient healing. These are the following steps in making this appliance, a) impression used alginate. Impression should be free of bubbles and voids on the teeth and palate. It should be poured immediately with die stone gypsum. When the stone is set, cast is withdrawn from the impression and should be free of bubbles and voids; b) excess stone on labial portion is trimmed to the depth of the vestibule. With a pressure or vacuum adapter, a 2-mm-thick, hard, clear acrylic resin sheet is adapted to the cast. Some products offer a dualsided acrylic resin sheet with a soft side for the teeth and a hard side on which to develop the occlusion. This $2.5 \mathrm{~mm}$ thick should be considered since it offers good retention and comfort during treatment; 3) the outline of the appliance is cut off the cast with separating disk. The cut is made at the level of the interdental papilla on the buccal and labial surface of the teeth. The posterior pa- 
latal area is cut along a straight line connecting the distal aspects of each second molar; d) appliance is then removed from the cast. Hard rubber wheel can be used to eliminate excess acrylic in the palatal area; $e$ ) the lingual border is extended 10-12 $\mathrm{mm}$ from the gingival border of the teeth. A large acrylic bur is used to smooth rough edges. The labial border is terminated between the incisal and middle thirds of the anterior teeth. (The border around the posterior teeth may be slightlylonger). It is safer to leave the border a little longer because if the appliance does not completely seat intraorally, the borders can be shortened until the appliance is fit; f) a small amount of clear self-curing acrylic resin is added to the occlusal surface of the anterior portion. This acrylic will act as the anterior stop, with the size approximately $4 \mathrm{~mm}$ wide and should extend to the region where a mandibular anterior central incisor will contact; g) evaluation is done intraorally. Appliance should fit, offering adequate retention and stability. Lip and tongue movement should not dislodge it. When pressure applied, it should not cause tipping or loosening. If the borders of the appliance have been maintained near the junction of the middle and incisal thirds on the facial surfaces of the teeth, adequate retention will be achieved; $h$ ) if it does not seat completely, it can be manipulated by heating it extra orally with a hair dryer and reseated on the teeth. This will help achieve a wellfitting appliance. Care must be taken not to overheat the plastic or all shape may be lost. ${ }^{5}$

For the stabilization appliance to be effective optimally, condyles must be located on their most musculoskeletally stable position, which is centric relation (CR). ${ }^{5}$ Two techniques have become widely used for finding $C R$, namely 1 ) bilateral manual manipulation technique. The bilateral manipulation suggested by Peter Dawson in 1974, is the method that has been largely utilized by those who adhere to functionally generated path techniques. They have suggested that the condyles do not always move superiorly, but sometimes, in response to posterior guidance from the operators, they move inferiorly. They emphasized the importance of superior placement of the condyles in the fossa when attempting to record CR. ${ }^{10}$

The bimanual manipulation technique was performed by placing both operator's hands under the subject's mandible. Slight superior pressure was applied at the gonial angle and inferior pressure at the mandibular symphysis, little effort is utilized in gaining a posterior placement. An attempt is made to obtain an arcing motion of the mandible in its most superior position guiding the mandible into registration material. This registration material was allowed to harden and removed from the subject's mouth. ${ }^{10,11}$

2) by placing a stopper on the anterior region of the appliance and the muscles are used to locate the musculoskeletally stable position of the condyles. This point is achieved by placing an occlusal stop in the anterior of the mouth and asking the patient to attempt to close on the posterior teeth. Without posterior tooth contacts, the elevator muscles will allow the condyles to be place into their musculoskeletally stable positions and it can be accomplished with a leaf gauge. The concept behind leaf gauge is when only the anterior teeth occlude (disengaging the posterior teeth) the directional force provided by the elevator muscles (temporalis, masseter, medial pterygoid) seats the condyles in a superoanterior position within the fossae. ${ }^{5}$

The anterior stop provided by the leaf gauge acts as a fulcrum, allowing the condyles to be pivoted to musculoskeletal position in the fossae. Leaf gauge must be used carefully so the condyle will not be deflected away from CR. If the leaf gauge is too rigid, it may provide a posterior slope deflecting the mandible posteriorly as the elevator muscles contract. Another mistakes may result if patient attempts to bite on the leaf gauge in a slightly forward position as this will lead to protruding of the mandible from the CR position. ${ }^{5}$

\section{Anterior positioning appliance}

The anterior positioning appliance is an interocclusal device that encourages the mandible to assume a position more anterior to the intercuspal position. It may be useful for the management of certain disc derangement disorders since anterior positioning of the condyle may help provide a better condyle-disc relationship, thus allowing better opportunity for tissue adaptation or repair. This appliance is used to temporarily position the condyles more anterior then their musculoskeletally stable position to relieve the existing symptoms. .,12 $^{12}$

Like the stabilization appliance, the anterior positioning appliance is a full-arch hard acrylic device that can be used in either arch. However, maxillary arch is preferred because a guiding ramp can be more easily fabricated to direct the mandible into the desired forward position. The steps in fabricating anterior positioning appliance are

a) the appliance does not extend onto the labial surfaces of the teeth and hence is more esthetic than the stabilization appliance; b) the key to suc- 
cessful construction of the appliance is in locating the correct anterior stop. It is made on the anterior part of the appliance by asking the patient to protrude his mandible and finding the position in which the clicking and the joint pain is relieved; c) sometimes, stoppage of the clicking alone does not indicate that the mandible is positioned anterior to the retrodiscal tissue. So, if symptoms fail to resolve, more advanced methods like arthroscopy and MRI may be used to ascertain the position of the mandible. ${ }^{5,12}$

\section{Anterior bite plane}

The anterior bite plane is a hard acrylic appliance worn over the maxillary teeth providing contact with only the mandibular anterior teeth. This appliance is intended to disengage the posterior teeth and thus eliminate their influence on the function of the masticatory system. Bite plane therapy should be used when there's a muscle disorder caused because of excessive loading of the musculature and hyper occlusion; this bite plate allow muscle to relax. It is suggested mostly in patients having muscle pain which could be acute/ chronic. The appliance has been suggested for the treatment of muscle disorders related to orthopedic instability or an acute change in the occlusal condition. Parafunctional activity may also be treated with this but only for short periods of time..$^{5,8}$

\section{Posterior bite plane}

It is a hard acrylic appliance, which covers the posterior mandibular teeth and is usually fabricated for mandibular teeth and connected by a cast lingual bar. The objective of this appliance is to bring about changes in the vertical dimension and the mandibular positioning. This appliance has been advocated in cases of severe loss of vertical dimension or when there is a need to make major changes in anterior positioning of the mandible. The use of this device may be helpful for certain disc derangement disorders, although it has not been studied well for this condition. . $^{5} 12$

\section{Pivoting appliance}

The pivoting device is fabricated with hard acrylic resin that covers the maxillary or mandibular arch with a single posterior occlusal contact, placed as far posteriorly as possible, in each quadrant. When superior force is applied under the chin, the tendency is to push the anterior teeth close together and pivot the condyles downward around the posterior pivoting point. This appliance was originally developed with the idea that it would reduce interarticular pressure and thus unload the articular surfaces of the joint. This was thought to be possible when the anterior teeth moved closer together, creating a fulcrum around the second molar, pivoting the condyle downward and backward away from the fossa. This appliance was suggested for patients with internal derangements or with osteoarthritis. Unfortunately, a potential adverse effect with the use of this modified appliance may cause occlusal changes as a posterior open bite in pivot area. ${ }^{5,13}$

\section{Soft or resilient appliance}

It is fabricated from resilient material and is usually adapted to the maxillary teeth. The main aim is to achieve even and simultaneous contact with the opposing teeth. This appliance is worn during nighttime only. They can provide relief within 6 weeks. The goal of this treatment is to achieve even and simultaneous contact with the opposing teeth. In many cases, this is difficult to accomplish since most of the soft materials are difficult to precisely adjust. The most common indication is as a protective device for persons likely to receive trauma to their dental arches. Protective athletic splints decrease the likelihood of damage to the oral structures when trauma is received. Soft appliances have also been recommended for patients who exhibit high levels of clenching and bruxism. ${ }^{5,14}$

\section{Cognitive behavioral therapy}

Patients with chronic TMD usually present associated psychological factors that should be managed with specific interventions. This therapy is one of the treatments proposed to manage patients' thoughts, behaviours, and or feelings that might exacerbate pain symptoms. It is a non-invasive therapy and unlikely to have adverse effects. This can be in the form of lifestyle counseling, relaxation therapy, hypnosis, and biofeedback. $^{2,6}$

\section{Psychotherapy}

Occasionally TMD maybe the somaticexpression of an underlying psychological or psychiatric disorder such as depression or a conversion disorder. Clues to this possibility are when strange symptoms are reported, the patient demonstrates odd behavior, or the patient's suffering appears to be excessive or persistent beyond what would normally be expected of the condition itself. In these cases, psychiatric referral is a mandatory part of the overall management strategy. ${ }^{6}$ 


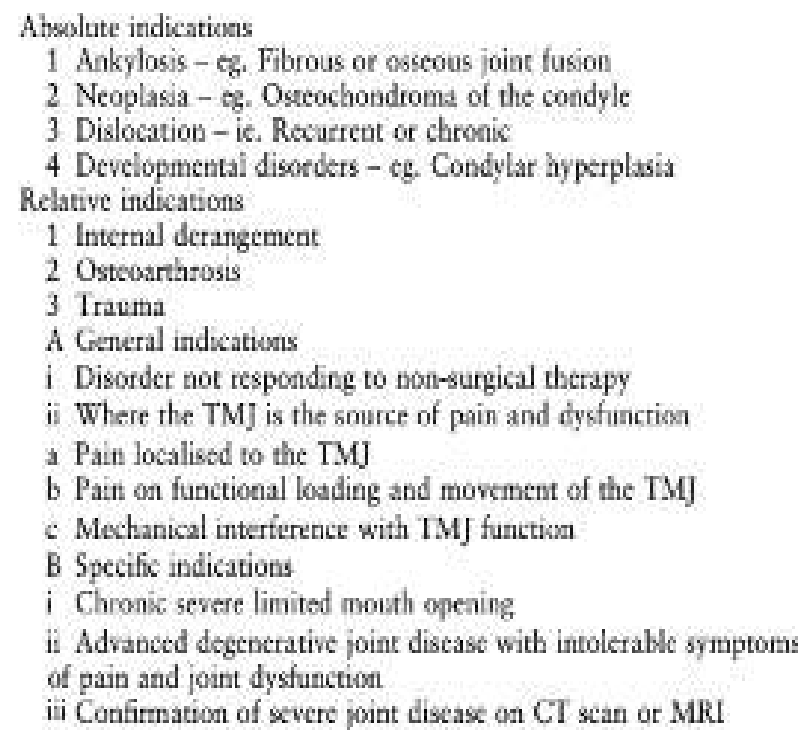

Figure 5 Indications for surgical treatment of the TMJ. The types of surgical procedures that can be performed on TMD are a) closed procedures; namely TMJ arthrocentesis and TMJ arthroscopy, and $\mathbf{b}$ ) open procedures; namely TMJ arthrotomy/arthroplasty and TMJjoint replacements.

\section{Other}

Acupuncture, botox injections, chiropractic/ osteopath jaw manipulation and other treatments have been tried in the management of TMD but have not been accepted in general practice due to lack of evidence in the effectiveness of these the- rapies. Stimulation of specific areas (or acupuncture points) causes the release of endogenous opioids (endorphins and enkephalins), which reduce pain sensation by flooding afferent interneurons with subthreshold stimulation. This effectively blocks the transmission of noxious impulses and thus reduces the sensation of pain. Intermittent stimulation of about two pulses per second appears to be effective in reducing discomfort associated with masticatory dysfunction. ${ }^{3,6}$

\section{TMJ surgery}

Specific indications for TMJ surgery include severe and chronic limited mouth opening and severe mechanical disturbances such as painful clicks and crepitus that fail to respond to nonsurgical measures. ${ }^{5}$

Treatment for TMD is categorized into two types, namely definitive care and supportive care. The main goals of treatment for TMD are to reduce or eliminate pain and or joint noises, and to restore normal mandibular function. Dentist must learn to correctly diagnosis and properly treat the acute orofacial pain condition with practical and evidence-based approach. Acute pain management is necessary to prevent an acute condi-tion from becoming a chronic pain disorder in the future.

\section{REFERENCES}

1. Conville RM. The management of temporomandibular disorders: a headache in general practice. $\mathrm{Br} \mathrm{J}$ Gen Pract 2019; 69: 523-4.

2. Gil-Martinez A. Management of pain in patients with temporomandibular disorder (TMD): challenges and solutions. J Pain Res 2018;11: 571-87.

3. Collins T. Temporomandibularjoint disorders. InnovAiT 2020; 13(8): 475-83.

4. Kaur H. Prosthodontic management of temporomandibular disorders. J Indian Prosthodont Soc 2013; 13(4):400-5.

5. Okeson JP. Management of temporomandibular disorders and occlusion $8^{\text {th }}$ ed. Missouri: Elsevier; 2020

6. Dimitroulis G. Management of temporomandibular joint disorders: A surgeon's perspective. Aust Dent $\mathrm{J}$ 2018; 63(1): 79-90.

7. Xu GZ. Low-level laser therapy for temporomandibular disorders: a systematic review with meta-analysis. Pain Res Management 2018: 1-13.

8. P. Dhannawat. Different types of occlusal splint used in management of temporomandibular joint disordersa review. Eur J Mol Clin Med 2020; 7(7): 1787-94.

9. Jean-Francois C. Usefulness of occlusal splints. J Dentofac Anom Orthod 2012; 15: 204-15.

10. Duggal N. Meta-analysis of various methods of recording centric jaw relation- a literature review. Int J Health Sci Res 2017; 7(3): 341-50.

11. Jilani S. A simplified method of centric relation record. Int J Dent Oral Health 2018; 4(6):77-83.

12. Jain S. Occlusal appliance therapy : a review. Indian J Dent Sci 2015; 3(7): 98-102.

13. Alqutaibi AY. Types of occlusal splint in management of temporomandibular disorders (TMD). J Arthritis 2015; 4(4): 176-80.

14.Jankar AS. Occlusal splints: an innovative treatment modality in temporomandibular disorders. J Prosthodont Dent 2020; 15(2): 35-41. 